\title{
2-D contour perception resulting from kinetic occlusion
}

\author{
GEORGE J. ANDERSEN and JAMES M. CORTESE \\ University of Illinois at Urbana-Champaign, Champaign, Illinois
}

\begin{abstract}
Kinetic occlusion, the progressive deletion or accretion of texture elements as one surface covers or uncovers another, has been shown to be an important source of information for determining depth order. In the present study, the importance of this information for 2-D contour perception was examined. In Experiment 1, subjects were asked to discriminate four different target shapes defined solely by kinetic occlusion. Discrimination increased with an increase in texture density and velocity, with density as the major factor. In Experiment 2, the targets were defined by static untextured regions as well as by kinetic occlusion. Overall, accuracy was similar to that found in Experiment 1, indicating that the presence of static information had little impact on accuracy. In Experiment 3, subjects were unable to discriminate among the four targets when presented with static versions of the displays used in Experiment 2. The results from these experiments indicate that kinetic occlusion can be used for discrimination of different 2-D shapes and that density has a more important role in determining accuracy than velocity.
\end{abstract}

Kinetic occlusion, the progressive accretion or deletion of texture elements on one surface as it is covered or uncovered by another surface, has been shown to be an important source of information for determining the order of textured surfaces in depth (Andersen \& Braunstein, 1983; Braunstein, Andersen, \& Riefer, 1982; Kaplan, 1969; Yonas, Craton, \& Thompson, 1987). In one of the earliest studies on occlusion, Kaplan (1969) had subjects view displays in which two texture patterns, moving horizontally at different speeds, merged at a vertical margin. Both surfaces underwent deletion of texture elements at the margin. The subjects were asked to indicate which of the two texture fields appeared to be closer and which appeared to be farther away. The texture pattern that had the greater rate of deletion was usually perceived as the farther surface. More recent research has shown that kinetic occlusion can be used to disambiguate depth along the line of sight for orthographic projections of rotating transparent spheres (Andersen \& Braunstein, 1983; Braunstein et al., 1982), that the relative motion between a texture field and contour in kinetic occlusion displays is sufficient for the recovery of sign of depth (Yonas et al., 1987), and that occlusion is important for a veridical perception of point-light walker displays (Proffitt, Bertenthal, \& Roberts, 1984).

Gibson, Kaplan, Reynolds, and Wheeler (1969) considered the information from occlusion to be a perceptual form of object permanence. They argued that the appear-

This research was supported by NSF Grant BNS 8607217, awarded to George J. Andersen, and a Graduate Fellowship from the University of Ilinois, awarded to James M. Cortese. The authors would like to thank two anonymous reviewers for comments on an earlier draft. Reprint requests should be sent to George J. Andersen, Department of Psychology, University of Ilinois, 603 E. Daniel St., Champaign, IL 61820. ance or disappearance of texture from kinetic occlusion is different from the appearance or disappearance of objects that go out of existence or come into existence. Therefore, they proposed that different optical transformations must underlie these events if we are able to distinguish between them.

There is a second type of perceptual information that is available from occlusion. The accretion and deletion of texture elements can be used to determine the 2-D edge or contour of the occluding object. The perception of a contour from kinetic occlusion can be considered to be a dynamic example of an implicit or subjective contour recovered from occlusion. This information might be used for recognizing objects by part boundaries (Hoffman \& Richards, 1984) or object components (Biederman, 1985), and it would provide information that is mathematically sufficient for determining the 3-D shape (the regions of hyperbolic and elliptical curvature) of the occluding object (Koenderink, 1984).

Indeed, several recent studies have examined the usefulness of kinetic occlusion for the perception of subjective contours. Prazdny (1986) presented subjects with displays in which two inducing rectangles rotated behind an implicit triangle. Portions of the rectangles were occluded by the triangle during rotation. In one condition, the inducing rectangles were filled with static random dots while the surround (and hence the illusory figure) was filled with dynamic "snow" noise (the elements of the surround were not correlated across time). The subjects in this condition did not report a perception of an illusory figure. However, when these two random dot patterns were reversed (snow noise in the inducing rectangles and static random dots in the surround), the subjects reported a perception of the illusory figure. This suggests that subjective contours may be defined by global motion discon- 
tinuities and do not require the luminance boundaries present in displays used in previous studies (Bradley \& Lee, 1982; Kellman \& Cohen, 1984; see Kellman and Loukides, 1987, for other examples of subjective contour perception from kinetic occlusion).

An important issue that has not been examined by previous research consists of the conditions for the perception of the shape of an occluding object. Kaplan (1969) proposed that the rate of accretion and deletion was critical for determining the order of surfaces in depth. Two optical variables are important in determining this rate when the display contains no luminance-defined edge or boundary. First, the texture densities of the background and foreground objects are important. Given a constant rate of horizontal translation (perpendicular to the line of sight) of the object or background, the rate of accretion and deletion is proportional to the texture density of the background surface. A second important variable is the rate of horizontal translation. If the texture density of the background surface is held constant, then the rate of accretion and deletion would be proportional to the rate of translation.

The roles of these two variables suggest an important interaction: a change in one factor and an inverse proportional change in the other factor will result in a constant rate of accretion and deletion. If this rate is important for discriminating different contours then accuracy should be approximately the same when this rate is kept constant across different combinations of texture density and velocity.

Finally, a third variable that is likely to be important in determining the shape of an occluding object, although Kaplan (1969) did not discuss it, is the presence of changes in orientation of the edge or contour along which occlusion and disocclusion take place. When a low rate of accretion or deletion is present, the visual system might define the edge as a linear contour by interpolating between regions where accretion and deletion of texture has occurred. This type of analysis has been proposed for static subjective contours (Kellman \& Shipley, 1988). For a shape with linear edges orthogonal to the direction of motion, such as a square, this type of analysis would favor the correct recovery of the contour as well as the correct identification of the shape. Shapes that have variations in the orientation of the edges (with respect to the direction of motion) would be more difficult to identify, if the visual system interpolates linearly between regions of accretion and deletion. A shape with a single change in the orientation of the edge (such as a diamond shape) might be somewhat more difficult to identify, whereas a shape with multiple changes (such as a star shape) or continuous changes (such as a circle shape) is likely to be even more difficult to identify than a shape with linear edges.

\section{EXPERIMENT 1}

Our purpose in Experiment 1 was to determine the importance of velocity, density, and type of contour for discrimination of 2-D contours during kinetic occlusion. The
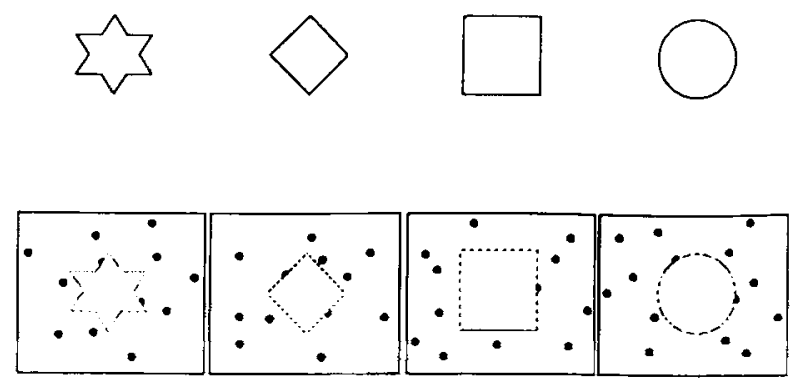

Figure 1. The four target shapes used in the present study. The lower figures represent the occlusion conditions examined in Experiments 2 and 3.

subjects viewed displays that simulated horizontal motion of a background texture field in relation to a static foreground target. Three variables were examined: the texture densities of the target and background, the rate of translation of the background, and the contour of the target. Four different targets were examined: a square, circle, diamond, and star (see Figure 1).

\section{Method}

Subjects. The subjects were 12 students from the University of Illinois, who were paid for their participation. All were naive regarding the purpose of the study, and all had normal or corrected-tonormal vision.

Design. Three independent variables were examined: the densities of the foreground and background fields of random dots (four levels), the velocity of the field of random dots (four levels), and the shape of the target figure (circle, square, diamond, or star), for a $4 \times 4 \times 4$ design.

Stimuli. The displays simulated horizontal translation towards the right or left of a random dot background relative to a static foreground figure. Whenever a background dot fell within the contour of the static foreground figure it was not plotted. The dot density of the foreground figure was equal to the density of the background field. Thus, the contour of the foreground figure was defined solely by the accretion and deletion of background texture.

Each figure had a height and width of $5.9 \mathrm{~cm}$, which at a viewing distance of $1.7 \mathrm{~m}$, corresponded to a visual angle of $2.0^{\circ}$. The condition of equal heights was necessary to establish equal rates of occlusion (which can be computed from the formula: rate of occlusion $=$ density of points $\times$ velocity of points $\times$ height of the figure).

The background dots fell into a region of approximately $156 \mathrm{~cm}^{2}$. The four projected density conditions were 10, 20, 40, or 80 dots, corresponding to $.064, .128, .256$, or .512 dots per square centimeter. The four velocity conditions were $.8,1.6,3.2$, and $6.4^{\circ} / \mathrm{sec}$. The display duration for each condition was $5.2 \mathrm{sec}$. The interstimulus interval was $4.0 \mathrm{sec}$.

Apparatus. The stimuli were displayed on a Hewlett-Packard Model 1310B X-Y display with a P-31 phosphor, controlled by a PDP-11/73 computer. The stimuli were refreshed at $30 \mathrm{~Hz}$. The subjects viewed the displays through a tube that limited the field of view to a circular area $8.5^{\circ}$ in diameter. The eye-to-screen distance was $1.7 \mathrm{~m}$. A black posterboard with white dots randomly positioned on the surface of the board, and a circle, square, diamond, and star figure cut out of posterboard material were used to illustrate the displays.

Procedure. The subjects participated individually. They were instructed that they would be viewing a series of displays consisting of a field of points moving behind a figure in the center of the display, and that the figure would be a circle, square, diamond, or 
star. Simulated examples of the displays were demonstrated with the hand-held model. The subjects were instructed that one of the four shapes would always be present, and that on each trial, they would be required to indicate verbally which shape they saw.

The subjects viewed each of the 64 displays $(4 \times 4 \times 4$ design) four times, for a total of 256 trials. The order of presentation was randomized. Eight sample displays that were shown first served as practice trials. At the end of the experiment, the subjects were asked debriefing questions, including, "Was there any particular strategy you used in making your response?"

\section{Results and Discussion}

For each subject, the average number of correct responses was computed for each condition. A three-way (density $\times$ velocity $\times$ target) analysis of variance (ANOVA) was conducted. A significant main effect $[F(3,33)=71.85, p<.001]$ was found for the density of the display. The mean percentage correct for the 10-, $20-, 40-$, and 80 -dot density levels were $40.73 \%, 63.79 \%$, $82.41 \%$, and $92.44 \%$. Post hoc comparisons (Tukey's hsd test) indicated significant differences between all pairs of the four levels, $p<.05$. The main effect for the velocity of the background was also significant $[F(3,33)=$ $42.58, p<.001]$. The mean percentages correct for the $.8,1.6,3.2$, and $6.4 \% \mathrm{sec}$ velocity levels were $60.41 \%$, $67.96 \%, 74.21 \%$, and $76.82 \%$, respectively. Post hoc comparisons indicated significant differences between all pairs of the four levels $(p<.05)$ with the exception of the $3.2 \% \mathrm{sec}$ and the $6.4 \% \mathrm{sec}$ conditions. There was also a main effect for the type of target $[F(3,33)=21.74$, $p<.01]$. The mean percentages correct for the circle, square, diamond, and star targets were $58.98 \%, 83.19 \%$, $77.99 \%$, and $59.24 \%$. Post hoc comparisons indicated significant differences between all pairs of the four target conditions $(p<.05)$, with the exception of the circle and star targets.

There were also significant two-way interactions between density and velocity $[F(9,99)=2.03, p<.05]$, density and target type $[F(9,99)=3.99, p<.01]$, and velocity and target type $[F(9,99)=2.71, p<.01]$. The interaction between density and velocity is shown in Figure 2. According to this interaction, discrimination accuracy increased with increased dot density at a faster rate for the high-velocity conditions than for the low-velocity conditions. The three-way interaction of density, velocity, and target type was not significant $[F(27,297)<1]$.

The $\omega^{2}$ values for the main effects of density, velocity, and target type were $.349, .036$, and .103 , respectively. The $\omega^{2}$ values for the two-way interactions between density and velocity, density and target type, and velocity and target type were $.003, .018$, and .007 , respectively.

The results indicate that increased density or velocity of the occlusion display resulted in an increase in discrimination of the four targets. Although the overall trends suggest that the rate of accretion and deletion was important, the greater variance accounted for by the density variable suggests that density was a more important factor in determining the discrimination.

A separate analysis was conducted to determine whether equivalent levels of accuracy in discriminating the four

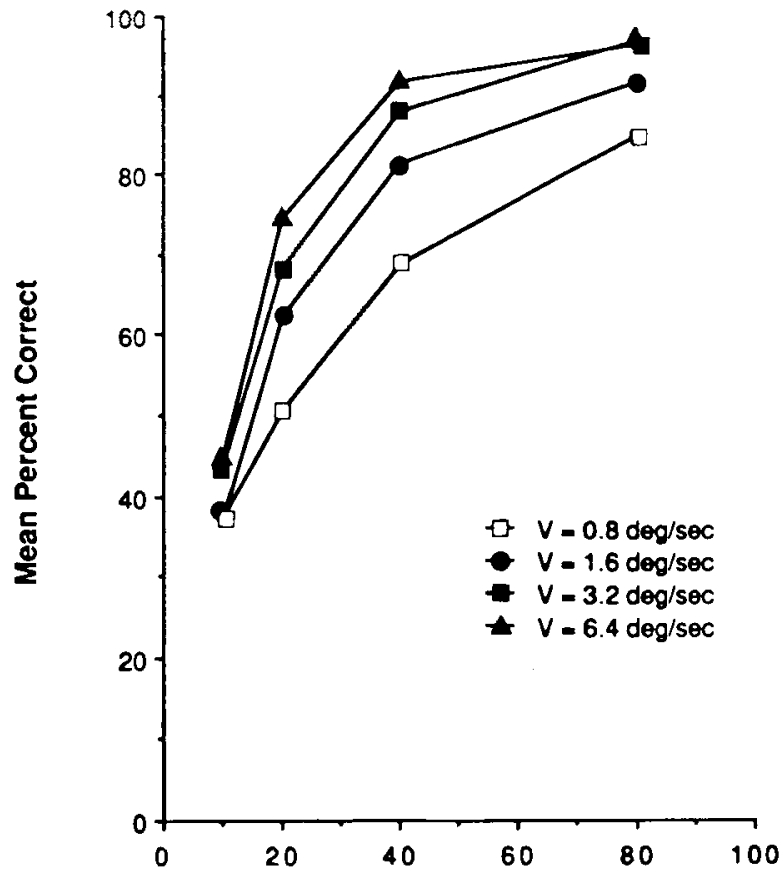

Number of Background Texture Elements

Figure 2. Interaction of the density of the background field with the velocity of the background field. The results are based on purely kinetic occlusion displays.

targets occurred for equivalent rates of accretion and deletion. One method of addressing this question is to determine whether the slopes for discrimination across conditions with equal rates of accretion and deletion were significantly different from zero. Consider the display with a density of 20 and a velocity of $.8 \% \mathrm{sec}$ and the display with a density of 10 and a velocity of $1.6 \% \mathrm{sec}$. The rates of accretion or deletion for these two displays are equal. Thus, if discrimination was based on this rate, then the accuracy of discrimination should have been approximately equal. This suggests that the slope between these two points should be zero. A $t$ test was conducted for the slopes of 12 pairs of data points representing the total possible combinations of constant rates of accretion and deletion. The mean of these slopes was significantly different from zero $[t(11)=5.17, p<.01]$, indicating that equivalent rates of accretion and deletion do not result in equal levels of accuracy.

An additional analysis was conducted on the errors that occurred. A confusion matrix (see Table 1) was computed to determine any trends that might exist concerning which targets were more readily confused with which other targets. The results indicate that the circle and square targets were more often mistaken for each other, whereas the diamond and star were more often mistaken for each other.

It was proposed that targets that contained either several changes in the orientation of edges along which accre- 
Table 1

Confusion Matrix for the Four Target Shapes

\begin{tabular}{ccccc}
\hline & \multicolumn{4}{c}{ Target } \\
\cline { 3 - 5 } Response & Circle & Square & Diamond & Star \\
\hline Circle & & & & \\
Experiment 1 & 58.98 & 10.03 & 7.42 & 8.72 \\
$\quad$ Experiment 2 & 62.41 & 10.76 & 9.46 & 7.47 \\
Square & & & & \\
$\quad$ Experiment 1 & 16.54 & 83.20 & 5.47 & 7.55 \\
$\quad$ Experiment 2 & 19.71 & 81.68 & 7.21 & 8.51 \\
Diamond & & & & \\
$\quad$ Experiment 1 & 17.08 & 3.39 & 77.99 & 24.48 \\
Experiment 2 & 11.02 & 4.34 & 69.10 & 23.52 \\
Star & & & & \\
Experiment 1 & 7.42 & 3.39 & 9.11 & 59.24 \\
Experiment 2 & 6.86 & 3.21 & 14.24 & 60.50 \\
\hline
\end{tabular}

Note-Values represent mean percentage of responses.

tion and deletion of texture occurred (such as a star) or a continuous change in edge orientation (such as a circle) would be more difficult to identify than targets with a single orientation change (such as a diamond) or no orientation change (such as a square). Indeed, the subjects were significantly less accurate in identifying the star and circle targets than in identifying the diamond and square targets. These results suggest that orientation changes in the edges that define an object may be important for discrimination of 2-D shapes on the basis of kinetic occlusion and that a greater number of orientation changes are likely to result in decreased accuracy in discrimination of the shapes.

\section{EXPERIMENT 2}

In Experiment 1, subjects discriminated four different foreground figures on the basis of kinetic occlusion. Under these conditions, the presence of an edge or contour was only perceived when the texture pattern of the background was accreted and deleted over time. An alternative type of kinetic occlusion occurs when the accretion and deletion occurs over time, but the boundaries of object and background are also distinguishable on the basis of different texture densities. In this situation, static information is available. An extreme case of this difference occurs when the foreground object has no texture. In Experiment 2 , the subjects viewed displays that were similar to those used in Experiment 1, except that the foreground object did not contain texture.

There are several reasons why it is important to examine this type of occlusion. First, because of perspective, it is common in the visual world to have different texture densities for background and foreground objects. Such information has been shown to be important for segmentation of boundaries (see Beck, 1982; Bergen \& Julesz, 1983; Pashler, 1988; Treisman \& Gelade, 1980). Second, there are many cases in the real world in which ambient light conditions result in a back illuminated silhouette of a foreground object. In this instance, there may be no texture visible on the foreground object. Finally, most displays utilized in research on the perception of subjective contour are cases in which a texture pattern is not present on a foreground figure. Dynamic displays in which no texture is present on the foreground figure can be considered to be dynamic cases of the types of static displays used in research on subjective contours. (See Parks, 1984, for a review of the research on subjective contours.)

In Experiment 2, the subjects viewed displays in which the foreground object was not covered by a texture pattern. Instead, it was demarcated by a textureless region in the display (see Figure 1). During motion of the background, the texture elements on the background disappeared and reappeared as they were projected behind the foreground target. The texture density of the background, the velocity of the background, and the type of foreground target were examined.

\section{Method}

Subjects. The subjects were 18 University of Illinois undergraduate students from an introductory psychology course, who were given course credit for their participation. All were naive as to the purpose of the study, and all had normal or corrected-to-normal vision.

Design. The three variables in this experiment were density of the field of dots, velocity of the field of dots, and shape of the target figure (circle, square, diamond, or star).

Stimuli. The stimuli were exactly the same as in Experiment 1, with the exception that the stationary dots on the foreground figure were not plotted.

Apparatus. The same apparatus as that in Experiment 1 was used.

Procedure. The procedure for this experiment was the same as that in Experiment 1.

\section{Results and Discussion}

The proportion of correct responses was computed for each subject for each condition and examined in a threeway (target $x$ velocity $\times$ speed) ANOVA. There was a significant main effect for the texture density of the display $[F(3,51)=262.7, p<.001]$. The mean percentages correct for the 10-, 20-, 40-, and 80-dot density levels were $40.79 \%, 60.32 \%, 80.12 \%$, and $92.88 \%$. Post hoc comparisons indicated significant differences $(p<.05)$ between all pairs of the four levels. There was also a significant main effect for the velocity of the display $[F(3,51)$ $=88.5, p<.001]$. The mean percentages correct for the $.8,1.6,3.2$, and $6.4 \%$ sec velocity levels were $52.5 \%$, $64.8 \%, 74.8 \%$, and $81.9 \%$. Post hoc comparisons indicated significant differences $(p<.05)$ between all four levels. The main effect for the type of target was also significant $[F(3,51)=20.4, p<.001]$. The mean percentages correct for the square, diamond, circle, and star were $81.86 \%, 69.0 \%, 62.7 \%$, and $60.6 \%$. Post hoc comparisons indicated significant differences $(p<.05)$ between all pairs of the four targets with the exception of the circle and star. The $\omega^{2}$ values of the density, velocity, and type of target main effects were $.336, .104$, and .056 , respectively.

There were also significant two-way interactions between the density and velocity $[F(9,153)=4.16$, $p<.01]$, between the density and type of target $[F(9,153)=2.72, p<.01]$, and between the velocity 
and type of target $[F(9,153)=2.46, p<.05]$. The $\omega^{2}$ values for these interactions were $.009, .007$, and .004 , respectively. The three-way interaction was not significant $[F(27,459)=1.38, p>.05]$.

The results from Experiment 2 are shown in Figure 3. The results were remarkably similar to the results obtained in Experiment 1, which indicated that both density and velocity were important variables for providing occlusion information. Of particular interest is whether the results could have been determined by the rates of accretion and deletion. A $t$ test (see Experiment 1) showed the mean of the slopes to be significantly different from zero $[t(11)=4.56, p<.01]$, indicating that equivalent rates of accretion and deletion do not result in equal levels of accuracy.

One interesting finding was the overall decrease in accuracy for displays in Experiment 2 as compared to Experiment 1. From comparison of the curves in Figures 2 and 3, it is clear that subjects were less accurate in correctly discriminating the four target figures in the second experiment. This is a surprising result, for the displays in Experiment 2 had static information that may have been useful in identifying the target. It is possible that this difference in accuracy occurred because different subjects were used in these two experiments. However, an alternative explanation is that the presence of two texture fields on either side of an occluding edge might facilitate discrimination of the edge. If texture is present on both sides

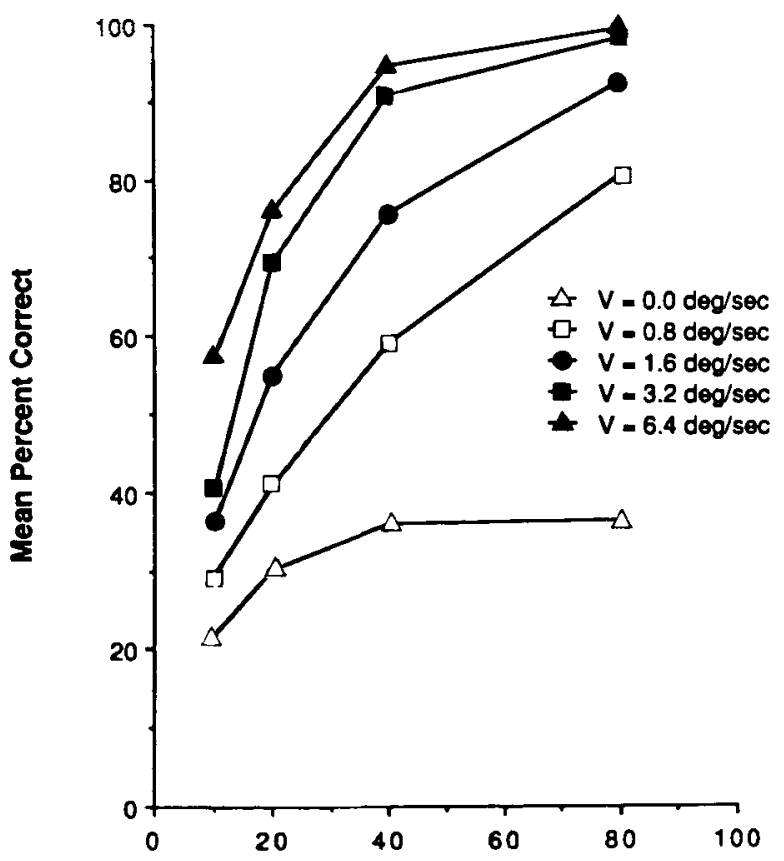

Number of Background Texture Elements

Figure 3. Interaction of the density of the background field with the velocity of the background field. The results are based on $\mathrm{dy}$ namic displays that contained static texture information. The lower curve is derived from the static trials examined in Experiment 3. of the contour, then it may be easier for the visual system to determine where accretion and deletion of texture (on the background object) and the preservation of texture (on the foreground object) is occurring in adjacent regions in the image. This type of information was available in the displays in Experiment 1, but it was not present in the displays in Experiment 2, in which texture was not present on the foreground object. Under these conditions, a subject may be able to determine the general form of the target contour from static information that is available (the lack of texture within a region of the image), but this information may be less useful than the presence of foreground texture for the determination of the exact location of edges.

A confusion matrix was computed for the errors in Experiment 2 (see Table 1). The results, which were similar to the results of the confusion matrix tabulated for Experiment 1 , indicate that the circle and square targets were more often mistaken for each other, while the diamond and star were more often mistaken for each other.

\section{EXPERIMENT 3}

In Experiment 1, the subjects were able to use kinetic occlusion information to discriminate targets when equal texture density was present on the foreground and background. In Experiment 2, the subjects also were able to use kinetic occlusion information to discriminate targets when the foreground was a textureless region. Indeed, the displays used in Experiments 1 and 2 resulted in similar rates of accuracy. The purpose of Experiment 3 was to determine whether a textureless region against a static background was sufficient for discrimination of the targets and whether discrimination accuracy increased after viewing dynamic display conditions.

The subjects were shown both static and dynamic versions of the displays used in Experiment 2. The stimuli were shown in three phases. In the first phase, the subjects were shown the static displays and asked to indicate which of the four targets was the foreground figure. In the second phase, the subjects were shown the dynamic displays used in Experiment 2, which had resulted in high rates of accuracy for discriminating the four targets. In the third phase, the subjects were shown the static displays again, and they were asked to make discrimination judgments. A comparison of the accuracy rates for the first and third phase will allow us to determine whether the viewing of dynamic displays from Experiment 2 improved overall accuracy.

\section{Method}

Subjects. The subjects were six undergraduate University of IIlinois students, who were paid for their participation. All six were naive regarding the purpose of the study, and all had normal or corrected-to-normal vision.

Design. The independent variables were the density of the display, the type of foreground target, and whether the subject viewed the display before or after the dynamic displays.

Stimuli. Two sets of stimuli were used. One set consisted of static versions of the displays used in Experiment 2. The other set of 
stimuli were dynamic displays identical to those used in Experiment 2

Apparatus. The same apparatus was used as in Experiment 1. Procedure. The subjects viewed each of the 16 static displays (four levels of density $\times$ four shapes) four times, in a random order, for a total of 64 trials. The subjects were then shown 64 dynamic displays, followed by another 64 trials of the static displays.

\section{Results and Discussion}

Dynamic trials. For each subject, the average number of correct responses was computed for each condition. A three-way (density $\times$ velocity $\times$ target) ANOVA was conducted. A significant main effect $[F(3,15)=52.68$, $p<.001]$, was found for the density of the display. The mean percentages correct for the 10-, 20-, 40-, and 80dot density levels were $45.6 \%, 62.9 \%, 80.6 \%$, and $94.4 \%$. Post hoc comparisons (Tukey's hsd test) indicated significant differences between all pairs of the four levels $(p<.05)$. The main effect for the velocity of the background was also significant $[F(3,15)=18.61, p<.001]$. The mean percentages correct for the $.8,1.6,3.2$, and $6.4 \% \mathrm{sec}$ velocity levels were $59.6 \%, 68.5 \%, 76.6 \%$, and $79 \%$, respectively. Post hoc comparisons indicated significant differences between all pairs of all four conditions with the exception of the $3.2^{\circ}$ and $6.4^{\circ}$ conditions $(p<.05)$. There was also a significant two-way interaction between density and velocity $[F(9,45)=2.76$, $p<.05]$. There were no other significant main effects or interactions. These results are similar to the results obtained in Experiment 2.

Static trials. The proportion of correct responses for each subject was computed and examined in a three-way (density $x$ target $\times$ before/after) ANOVA. The main effect for density was significant $[F(3,15)=5.23$, $p<.05]$. The average percentages correct for the 10-, 20 -, 40 -, and 80 -dot density levels were $21.87 \%, 30.2 \%$, $35.93 \%$, and $36.45 \%$. Post hoc comparisons indicated significant differences between all pairs of dot-density conditions with the exception of the 40 - and 80-dot density levels. The $\omega^{2}$ value for this effect was .026 . There were no other significant main effects or interactions.

The results are shown in Figure 3, along with the results from Experiment 2, for purposes of comparison. As can be seen in Figure 3, the overall level of accuracy for static trials was below the level of accuracy for the lowest velocity conditions. This occurred regardless of whether the responses were made before or after viewing the dynamic trials. These results indicate that the static displays were not effective in providing the information for contour perception that was provided in the dynamic conditions in Experiments 1 and 2. One exception to this result was found for the lowest density conditions for the circle target. On those trials, the subjects responded with greater accuracy for the static trials as compared with the dynamic trials. This result is most likely a response bias on the part of the subjects, since the lowest density levels for all three other figures were below chance levels $(25 \%)$.

\section{GENERAL DISCUSSION}

The results of the present experiments indicate that dynamic occlusion can be used for discrimination of different 2-D contours. In Experiment 1, the subjects' judgments of the target (defined by pure kinetic information) were primarily the result of the density of the display. In Experiment 2, each display simulated a silhouette of the foreground target without texture elements present. The subjects' responses were again based primarily on the density of the display. Experiment 1, without static information present, and Experiment 2, with static information available, produced very similar results. This suggests that the static information present in the dynamic displays in Experiment 2 was not useful. Indeed, during debriefing, only 1 subject out of 12 reported paying any attention to the static texture elements. The results of Experiment 3 further support this conclusion. When the subjects were presented with displays without dynamic occlusion information, accuracy was close to chance levels.

The presence of orientation changes in the edges of the targets was an important factor in the first and second experiment. Targets that were expected to be more difficult to identify on the basis of the number of orientation changes relative to the direction of motion resulted in lower accuracy rates than targets with fewer orientation changes. The square target, whose contour was defined by vertical edges that contained no orientation change along the edges at which occlusion and disocclusion occurred, produced the highest accuracy. On the other hand, the star target, which contained several orientation changes, and the circle target, which contained a continuous change in orientation, produced the lowest levels of accuracy. The diamond target, which contained only one orientation change, had accuracy levels higher than the circle or star targets but lower than the square target. These results might reflect a bias toward using a minimum distance criterion to interpolate the contour between regions where accretion and deletion has occurred. This concept is not new; it has been proposed by Kellman and Shipley (1988) in explaining continuity effects with traditional subjective contours.

An additional explanation for different levels of accuracy in discriminating of the targets concerns the information available for defining the edge. There are two sources of information that can be used. One is the accretion and deletion of texture elements. However, a second source of information is a local shear component defined by differences in the velocities of texture elements located on either side of the implicit contour. Both sources of information can be used for the perception of image boundaries or contours (van Doorn \& Koenderink, 1983; the present study), and both have been proposed as being processed by different mechanisms (Richards \& Liberman, 1982). The availability of these two sources of information depends on the local orientation of the edge, 
relative to the direction of motion. If the edge is parallel to the direction of motion, then the difference in velocity between the foreground target and the background provides local shear information. Under these conditions, accretion and deletion of texture elements will not occur. On the other hand, if the orientation of the edge is orthogonal to the direction of motion, then accretion and deletion of texture elements will occur, but local shear will not be present. Variations in the orientation of the contour therefore represent changes in the availability of these sources of information. An important issue for future research would be to directly examine the relative importance of these two sources of information for edge detection from motion.

The present results indicate that the density of the display is critical for discriminating different contours on the basis of occlusion information. These results are similar to results obtained by van Doorn and her colleagues (van de Grind, Koenderink, \& van Doorn, 1986; van Doorn \& Koenderink, 1982, 1983), in which the signal-to-noise ratio was varied for displays simulating accretion and deletion of two adjacent flow fields. In their experiments, threshold values were derived for detection of the presence of linear edges defined by coherent patterns of motion. They proposed that Reichardt bilocal detectors may be responsible for determining the presence of a subjective contour defined by motion. It is also reasonable to propose that bilocal detectors are important for detecting more complex contours, such as those examined in the present study.

\section{REFERENCES}

Andersen, G. J., \& Braunstein, M. L. (1983). Dynamic occlusion in the perception of rotation in depth. Perception \& Psychophysics, 34, 356-362.

BECK, J. (1982). Texture segmentation. In J. Beck (Ed.), Organization and representation in perception (pp. 285-317). Hillsdale, NJ: Erlbaum.

Bergen, J. R., \& Julesz, B. (1983). Parallel versus serial processing in rapid pattern discrimination. Nature, 303, 696-698.

Biederman, I. (1985). Human image understanding: Recent research and a theory. Computer Vision, Graphics, \& Image Processing, 32, 29-73.

Bradley, D. R., \& LeE, K. (1982). Animated subjective contours. Perception \& Psychophysics, 32, 393-395.
Braunstein, M. L., Andersen, G. J., \& Riefer, D. M. (1982). The use of occlusion to resolve ambiguity in parallel projections. Perception \& Psychophysics, 31, 261-267.

Gibson, J. J., Kaplan, G. A., Reynolds, H. N., Jr., \& Wheeler, K. (1969). The change from visible to invisible: A study of optic transitions. Perception \& Psychophysics, 5, 113-116.

Hoffman, D. D., Richards, W. A. (1984). Parts of recognition. Cognition, 18, 65-96.

Kaplan, G. A. (1969). Kinetic disruption of optical texture: The perception of depth at an edge. Perception \& Psychophysics, 6, 193-198.

Kellman, P. J., \& Cohen, M. H. (1984). Kinetic subjective contours. Perception \& Psychophysics, 35, 237-244.

Kellman, P. J., L Loukides, M. G. (1987). An object perception approach to static and kinetic subjective contours. In S. Petry \& G. E. Meyer (Eds.), The perception of illusory contours. Secaucus, NJ: Springer-Verlag.

Kellman, P. J., \& SiPley, T. F. (1988, November). Interpolation processes in visual object perception: Evidence for a discontinuity theory. Paper presented at the 28th meeting of the Psychonomic Society, Seattle, WA.

KoENDERINK, J. J. (1984). What does the occluding contour tell us about shape? Perception, 13, 321-330.

PARKs, T. E. (1984). Illusory figures: A (mostly) atheoretical review. Psychological Bulletin, 95, 282-300.

PAshler, H. (1988). Cross-dimensional interaction and texture segregation. Perception \& Psychophysics, 43, 307-318.

Prazdny, K. (1986). Illusory contours from inducers defined solely by spatiotemporal correlation. Perception \& Psychophysics, 39, 175-178.

Profhtt, D. R., Bertenthal, B. I., Roberts, R. J., JR. (1984).

The role of occlusion in reducing multistability in moving point-light displays. Perception \& Psychophysics, 36, 315-323.

Richards, W., Lieberman, H. R. (1982). Velocity blindness during shearing motion. Vision Research, 22, 97-100.

Treisman, A., \&elade, G. (1980). A feature integration theory of attention. Cognitive Psychology, 12, 97.136.

van de Grind, W. A., Koenderink, J. J., van Doorn, A. J. (1986). The distribution of human motion detector properties in the monocular visual field. Vision Research, 26, 797-810.

van DoORn, A. J., Koenderink, J. J. (1982). Visibility of movement gradients. Biological Cybermetics, 44, 167-175.

van Doorn, A. J., Koenderink, J. J. (1983). The stnucture of the human motion detection system. IEEE Transactions of Systems, Man, \& Cybernetics, 13, 916-922.

Yonas, A., Craton, L. G., \& Thompson, W. B. (1987). Relative motion: Kinetic information for the order of depth at an edge. Perception \& Psychophysics, 41, 53-59.

(Manuscript received July 15, 1988; revision accepted for publication December 8,1988 .) 